\title{
Investigation of the physico-chemical and microstructure changes of beef meat during frozen storage at $-23^{\circ} \mathrm{C}$
}

\begin{abstract}
Saliha Lakehal ${ }^{*}$, Omar Bennoune ${ }^{1}$, Ammar Ayachi $^{1}$
A b s $t r$ a c $t$ : Freezing beef meat is the most effective way to extend its storage life. However, there is little information about whether this practice alters the microstructure of beef and its effects on meat quality. For this reason, the object of our research was to determine the effect of frozen storage (one year at $-23^{\circ} \mathrm{C}$, with meat examined every two months) on physical, chemical and microstructural properties of beef in cuts of 20 Biceps femoris muscles. Significant physical changes were detected at different frozen storage durations, including increases in $\mathrm{pH}$ and yellowing $\left(b^{*}\right)$, as well as decreases in water activity, lightness $\left(L^{*}\right)$, and redness ( $\left.a^{*}\right)$. In terms of chemical characteristics, the protein solubility in the beef reduced, but lipid oxidation (TBARS) values considerably rose with frozen storage duration. The width of ice crystals in frozen beef steadily increased as storage time was extended to 12 months, indicating structural changes in the frozen meat.
\end{abstract}

Keywords: beef, physico-chemical parameters, microstructure, freezing, storage time.

\section{Introduction}

Meat is an important part of a well-balanced and varied diet, since its nutritional components fulfil the demands of the majority of people for essential nutrients, including protein, minerals and vitamins (Rahman et al., 2015). The worldwide beef meat export industry is valued over US\$ 13 billion, and demand is growing at a rate of $3.5 \%$ each year (Leygonie et al., 2012a). Freezing is frequently used in the food industry to preserve perishable substances for lengthy periods of time (Wang et al., 2020). Freezing, in particular, is important for keeping meat safe, allowing the meat industry to tailor its production to customer demand, adapt meat output to processing rates, and export meat to all parts of the world. The freezing technique employed in meat preservation is mainly concerned with slowing the growth and proliferation of meat spoilage microorganisms as well as delaying other deleterious changes to colour, odour, texture, moisture and ultrastructure (Zhang et al., 2019).

As a result of freezing, ice crystals cause mechanical damage, structural changes and protein denaturation in meat (Jeong et al., 2011). Ice crystals disrupt the ultrastructure of meat and concentrate solutes, causing metabolic changes and influencing the meat's physical qualities (Leygonie et al., $2012 b)$. One of these changes is a reduction in water-holding capacity that reduces the tenderness and the overall eating quality of the meat, lowering its commercial value (Kim et al., 2015)frozen only, and 3 or 4 . weeks ageing at $-1.5^{\circ} \mathrm{C}$ then frozen. Protein oxidation also lowers meat quality by causing fragmentation or aggregation of proteins and diminishing protein solubility (Sun et al., 2002). Phospholipids containing a wide variety of fatty acids including polyunsaturated fatty acids are the lipid component of meat that is most sensitive to oxidation. As a result, radical secondary lipid oxidation can be produced during meat freezing (Medić et al., 2018), resulting in undesired alterations in meat quality (Deng et al., 2021). Therefore, researchers must have a complete understanding of the physicochemical and structural changes caused by frozen storage of meat (Bertram et al., 2007).

Numerous studies have reported on the impact of long-term preservation on physicochemical and biochemical changes in meat of different animal species (Muela et al., 2015; Medić et al., 2018; )although the influence of frozen holding temperatures was negligible. LL carbonyl, and nitrate and nitrite content responses were variable and yet broadly reflected an increased incidence of protein oxidation

${ }^{1}$ ESPA Laboratory, Department of Veterinary Sciences, Institute of Veterinary Science and Agricultural Sciences, Batna 1 University, Algeria.

*Corresponding author: Saliha Lakehal, lakehalsaliha@yahoo.fr 